\title{
Chebyshev-Legendre Spectral Approach in Fractional Integro-Differential Equations
}

\author{
Yousefi", E. Babolian, and S. Ghoroghi Shafiei \\ Faculty of Mathematical Sciences and Computer, Kharazmi University, Taleghani Avenue, Tehran, Iran; \\ A.yousefi12349@yahoo.com, E.babolian.34@gmail.com, s.ghorbani.shafiei@yahoo.com
}

\begin{abstract}
Objectives: In the current research we implement Chebyshev-Legendre spectral approach for a class of linear fractional integro-differential equations (FIDE) including those of Fredholm and Volterra types via initial conditions or two-point boundary value conditions. Methods: An equations derived that characterizes the approximation solution by using the shifted Legendre and shifted Chebyshev collocation points. By providing various numerical instances, the precision and efficiency of the developed algorithms were evaluated. We show that for a class of equations, we obtain the exact solutions. Results: Some particular linear solvers were presented for the linear and nonlinear FIDE with initial conditions by applying downshifted Legendre polynomials. The fractional derivatives were defined in the Caputo sense. Furthermore, a novel method developed by executing downshifted Legendre procedure in union with the downshifted Chebyshev collocation way for the solution of FIDE. Based on our research, the presented research is the first investigation regarding the Chebyshev-Legendre spectral approach for determining FIDE with initial conditions. Conclusion: In the current paper, A numerical algorithm was proposed to determine the overall equations, utilizing Gauss-collocation features and estimated the solution directly applying the downshifted Legendre polynomials.
\end{abstract}

Keywords: Chebyshev-Legendre Spectral Approach, FIDE, Spectral Method

\section{Introduction}

Consider the following FIDE

The initial conditions Where is the th-order derivative of are real constants and Moreover represents the Caputo fractional derivative of kind for the amounts define the boundary state of, and are given functions. In this paper, the other case will be deliberated. These kinds of equations (fractional Fredholm-Volterra integral) used in several modeling and design issues in mathematics mechanics, like dynamics of interfaces between nanoparticles and substrates and image signal process? Various investigation teams were investigated and stated on integro differential procedure and fractional differential procedure.

In global and local existence and uniqueness of answer for the FIDE of combined kind by considering the delay are determined.
In Equation (1), when, the equation decreases to a linear fractional differential one that the uniqueness and existence of answer were studied in . There are several research of the numerical answer of an integro-differential equation via fractional derivative when, for example collocation method by spline polynomial functions Taylor expansion method ${ }^{6}$, Homotopy analysis methode, investigated the application and employment of Adomian decomposition method to provide mathematical approximate solution.

Used Chebyshev wavelet for obtaining the numerical solution.

The current paper is conducted by the aim to yield fair answers of FIDE with the Caputo's fractional derivative. We use the Chebyshev-Legendre spectral method to obtain the solutions of these equationsto Present a collocation method based on shifted Applied a transferred Chebyshev collocation approach via the 
transferred Gauss -Chebyshev points. In 12 present an operational matrix for implementing Chebyshev spectral approach.

Considered the Chebyshev pseudo-spectral approach for finding numerical answer with the linear and nonlinear FIDE of Volterra kind ${ }^{\text {a }}$. Applied operational Tau method and obtained direct and precision solution in the special case (the answer form is polynomial).

We have organized the paper as follows:

Any fundamental descriptions and features of fractional calculus are conferred in section 2. In the next section, we describe Chebyshev-Legendre spectral method and then in sections 4 and 5, we apply this method to general FIDE of Fredholm and Volterra types. At last, we represent some numerical results in section 6. Eventually, the concluding points are presented in subdivision 7 .

\section{Basic Definitions}

In this stage, we present basic description and features of fractional calculus.

Definition: Let be the smallest integer greater rather or identical to (real), represents the fractional differential operator of kind in the presence of Caputo, define in where Some basic properties are listed below:

\section{Chebyshev-Legendre Spectral Method}

\subsection{Transmitted Chebyshev polynomials}

The Chebyshev polynomials were described on the range with the below recurrence equation: with and the transmitted Chebyshev polynomials are defined by presenting the variation of parameter. We supposed the transmitted Chebyshev polynomials be denote by satisfying the relation as and By these definitions we will have

where is Kronecker delta and and For any function we can express regarding transmitted Chebyshev polynomials as follows: where the parameter are presented as Here, we turn to the Gauss-Chebyshev quadrature. For standard Gauss-Chebyshev quadrature, let be a collection of Chebyshev-Gauss quadrature nodes and weights: By using the before mentioned selections, there are presented as: where is polynomial of degree less than or equal. We instantly switch to the discrete Chebyshev converts.
The converts could be conducted with a matrix-vector multiplication by operations. When we use Chebyshev polynomials, it could be conducted via operations by?

Given, suppose be its Lagrange polynomial relative to the Gauss-Chebyshev points, and it can be rewritten as: where are evaluated via the convertor as follows. It was obvious that forward convertor could be evaluated via employing there in operations.

\subsection{Shifted Legendre Polynomial}

Let be the normal Legendre polynomial of degree next the followings can be identified

- Three-term recurrence relative and the two Legendre polynomials described as

- The Legendre polynomial has the expansion

- Orthogonality such that

- Symmetry property

Hence, if is odd (resp. even) function then be an odd (resp. even).

- The boundary amounts of the derivatives

Now, if we express the transmitted Legendre polynomial of degree via then according to Eq., the mathematical presentation of the transmitted Legendre polynomials of degree is presented as

If we let then we can define the weighted space with the following inner product where Therefore, the corresponding norm is By using of the Eq., we have

The boundary values of the fractional order derivative of order are These relations, will be of main application after that.

\subsection{Description of Chebyshev- Legendre Galerkin Approach}

The significant priority of application Chebyshev polynomials is that the discrete Chebyshev transform could be conducted in operations via employing On the other hand, the discrete Legendre transform is expensive, and therefore the method of Shen is applied to reduce the cost of solving the corresponding system.

In fact, we denote, the interpolation convertor related to the Gauss-Chebyshev points. The variation via Gauss-Legendre quadrature is that the Chebyshev interpolation operator is utilized in this research to be replaced via the Legendre interpolation transformer. The essential and significant idea of this approach is the 
use of interpolation transformer related to the GaussChebyshev-Lobattopoints to evaluate the specified functions and the use of inner product of Legendre polynomials to evaluate the unknown function together. At last, the solution procedures essentially the same as Legendre method except that Chebyshev-Legendre transform, among the amounts of a function at the Gauss- Chebyshev-Lobattopoints and the parameter and factors of its Legendre expansion, were required instead of the Legendre convertors. With more detain survey, considering the amounts of the known functions at Gauss- Chebyshev-Lobattopoints, we determine the value of , that is solution of Equation (1) at the these points as follow:

- (Pre-computation) Determination the nonzero factors of coefficient matrices.

- Determine the Legendre coefficient of and at GaussChebyshev-Lobattopoints (forward ChebyshevLegendre convertor).

- Determine the right-hand vector from linear system and solve.

- Evaluate at Gauss-Chebyshev-Lobattopoints (backward Chebyshev-Legendre transform).

For a function, the shifted Legendre expansion is where and

In function, just the initial-term transmitted Legendre polynomials are regarded. We let

Theorem 3.3.1 suppose is defined in Equation (25) and then as is the 2 operational matrix of Caputo derivative which is presented as:

where

Consider that in the first rows are zero.

Now, we describe the Legendre-Gauss integration in the interval We denote by, respectively the nodes and weights of the standard integration on the range We suppose, are nodes and weights of the Legendre-Gauss integration at the interval then, we have

According to Equation (29) for any, collection of all polynomials of degree at,

\section{Volterra Equations of Integro- Differential via Fractional Derivative (FIDE)}

Here, we want to discuss about a class of Volterra integrodifferential equations with fractional derivative of the form where Via the assuming and using Legendre spectral approximation to Equation (31), we will have

Let us denote Next Equation could be presented as: According to this method, by replacing in Equation (32), the following equations are obtained: Let us denote, where

According to theorem 3.1.1, by replacing by we obtain

Then, employing of the orthogonality relative of transmitted Legendre polynomials Equaion and Equation, are presented explicitly as also, because of then, Here for evaluating the integral term in Equation, we can apply any quadrature rule. Here, we implement the GaussChebyshev method.

Using the above relations, Equation (35) can be written in the following matrix form

\section{Fredholm FIDE}

In the current section, presented research concentrated on a type of Fredholm FIDE kind subjected to where is constant, are specified functions and is an undefined one to be evaluated. We assume that Equation has a unique solution. Therefore if then by implementing ChebyshevLegendre spectral method for Equation we can easily obtain

Similarly, by assigning the matrices and by definitions in section 4, the entries of the are definited in Equation until Equation respectively and In the recently relation, it is easier to use of the standard Gauss-Legendre integral quadrature by shifted Legendre polynomial in the interval for expanding the expression.

Similarly, The linear system in Equation and Equation, could be resolved by forming explicitly the factorizing that by according of

\section{Numerical Examples}

We would use the spectral method to several FIDE (Volterra and Fredholm type) with a known solution.

Example 1. First regard the below fractional Volterra integro-differential equation: subjected to

The permanent answer of this equation is according to the present approach, we evaluate the numerical solution for the solution of described approach is:

Example 2. Next we discuss the Fredholm FIDE with a parameter6 where is selected like the permanent answer 
Table 1. Comparison of for and for example 4

\begin{tabular}{lccccc}
\hline $\mathbf{t}$ & Method in & Our method with & $\mathbf{t}$ & Method in & Our method with \\
\hline 0.0 & 1.00000000 & 1.00000000 & 0.0 & 1.00000000 & 1.00000000 \\
0.1 & 1.12713251 & 1.10402221 & 0.1 & 1.11574165 & 1.10726965 \\
0.2 & 1.27412871 & 1.24400051 & 0.2 & 1.25407050 & 1.24414053 \\
0.3 & 1.44419228 & 1.40495333 & 0.3 & 1.41822408 & 1.40495548 \\
0.4 & 1.64216876 & 1.59552240 & 0.4 & 1.61210580 & 1.59612614 \\
0.5 & 1.86980974 & 1.82003001 & 0.5 & 1.84034522 & 1.82219532 \\
0.6 & 2.13679076 & 2.09321156 & 0.6 & 2.10836127 & 2.09324142 \\
0.7 & 2.44531829 & 2.40854621 & 0.7 & 2.42242223 & 2.40908655 \\
0.8 & 2.80675219 & 2.78010074 & 0.8 & 2.78973070 & 2.78026674 \\
0.9 & 3.22745248 & 3.21324015 & 0.9 & 3.21854010 & 3.21344147 \\
1.0 & 3.71828183 & 3.71828183 & 1.0 & 3.71828183 & 3.71828183 \\
\hline
\end{tabular}

is In this example, we derive an approximately solution as follow:

Example 3. Next we suppose the upcoming Volterra fractional integro-differential equation:

And the status of $y$ as the following and choose so that the permanent answer of this equation is

Our approximate solution for this integro-differential equation is:

Example 4. Suppose the below linear fourth-order FIDE

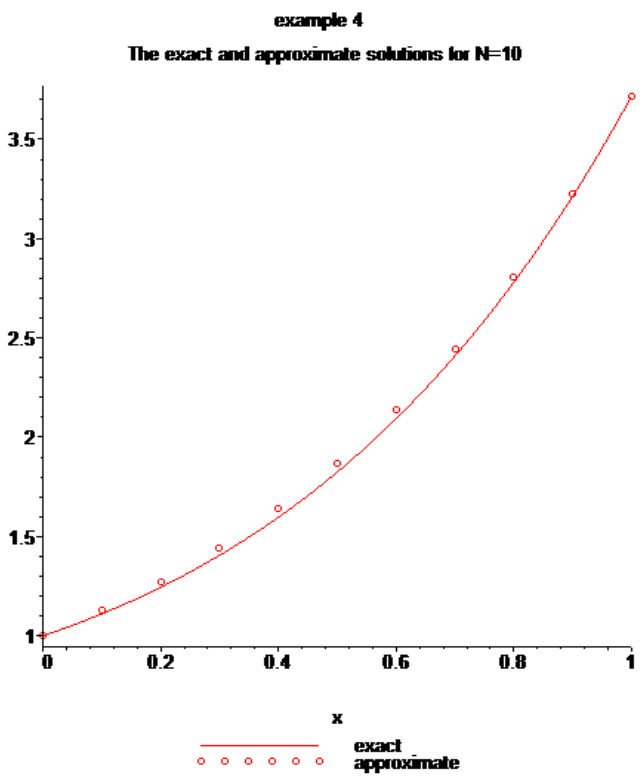

Figure 1. Describe the exact solution and approximate solution with for

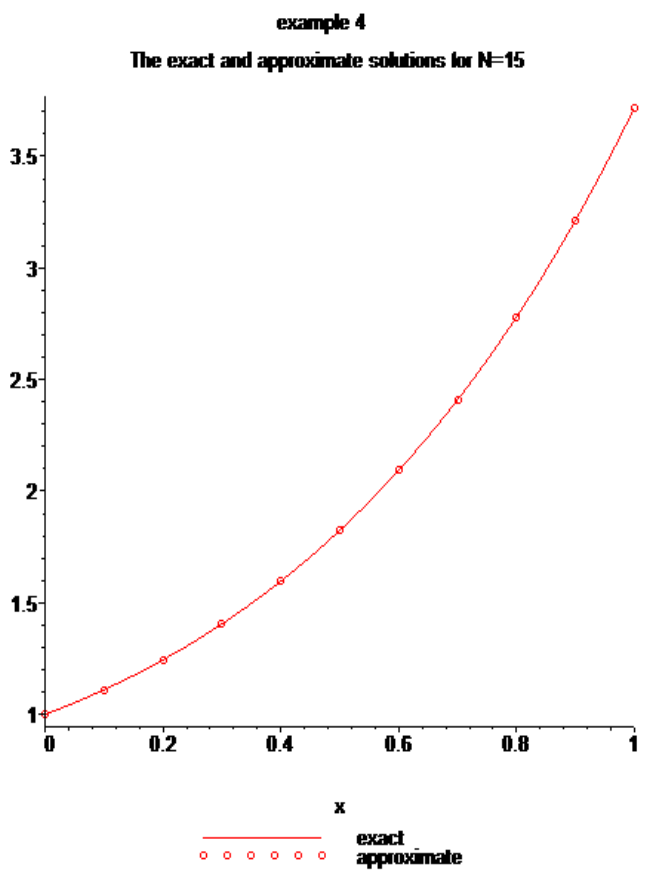

Figure 2. Describe the exact solution and approximate solution with for

In the instant of the permanent answer is. The manner of the permanent answer and evaluated answer of this instance while are given in Figures 1 and 2. To find the answer of this problem, we used the developed approach for via and for with. Finally the findings of proposed approach and those enhanced from Chebyshev pseudospectral method are given in Table 1 . 
Table 2. Comparison of for and and respectively for example 5

\begin{tabular}{cccccc}
\hline $\mathbf{t}$ & Method in & Our method with & $\mathbf{t}$ & Method in & Our method with \\
\hline 0.0 & 1.00000000 & 1.00000000 & 0.0 & 1.00000000 & 1.00000000 \\
0.1 & 1.10093503 & 1.10757565 & 0.1 & 1.10659794 & 1.10593045 \\
0.2 & 1.21465413 & 1.22583824 & 0.2 & 1.22402473 & 1.22281325 \\
0.3 & 1.34025419 & 1.35562954 & 0.3 & 1.35333811 & 1.35174095 \\
0.4 & 1.48271885 & 1.49842125 & 0.4 & 1.49577522 & 1.49396243 \\
0.5 & 1.63838198 & 1.65534854 & 0.5 & 1.65274868 & 1.65089025 \\
0.6 & 1.81382749 & 1.82825789 & 0.6 & 1.82585802 & 1.82411002 \\
0.7 & 2.00612089 & 2.01867254 & 0.7 & 2.01687273 & 2.01539021 \\
0.8 & 2.22060959 & 2.22898695 & 0.8 & 2.22777938 & 2.22669587 \\
0.9 & 2.45675040 & 2.46136754 & 0.9 & 2.46077210 & 2.46019578 \\
1.0 & 2.71828183 & 2.71828065 & 1.0 & 2.71828183 & 2.71828127 \\
\hline
\end{tabular}

In the method of fractional calculus, it is clear that while the fractional derivative aimed to, next the evaluated answer continuously wants to the permanent answer of the issue with derivative of. This follows from theorems 2.9 and 2.10. A detailed observer at the amounts enhanced by our method in Table 1, shows this fact. This is the case in the next example.

Example 5. Suppose the below nonlinear FIDE

In the example of the permanent answer is The manner of the permanent answer and evaluated one of this instance when are given in Figure 3. According to the example 4, we compare the evaluated answer for and with those obtained in Table 2.

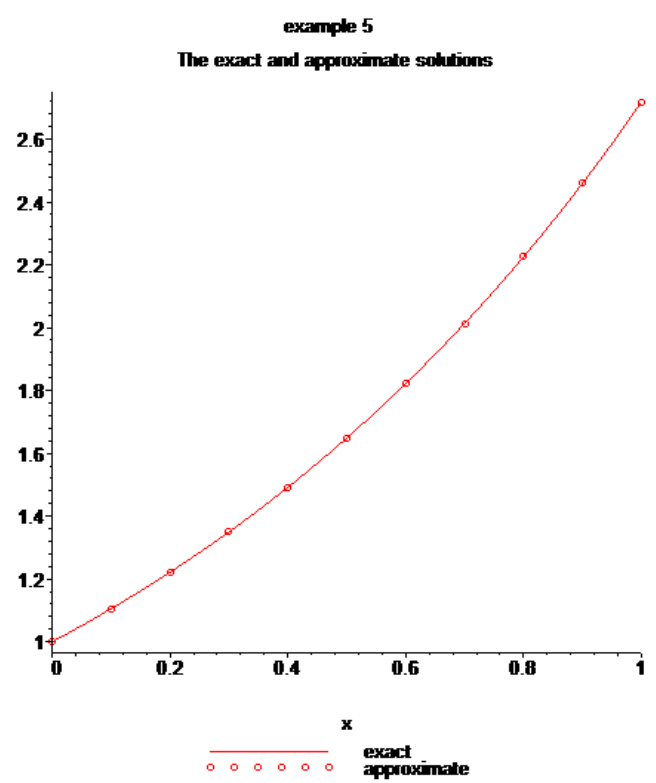

Figure 3. Describe the exact solution and approximate solution with for
Example 6. Suppose the nonlinear FIDE

In this problem, the function is selected such that the exact solution is By using the norm, we can see that the error norms are quite small. Figure 2 and 3 give satisfactory numerical performance in term of convergence.

\section{Conclusion}

In this research, we presented some actual direct solvers for the linear and nonlinear FIDE by initial status by utilizing transmitted Legendre polynomials. The fractional derivatives are reported in the Caputo presence. Furthermore, we produced a unique method performing downshifted Legendre system in joining by the transmitted Chebyshev set procedure for the statistical resolution of FIDE. According to our research, this is the original research regarding the Chebyshev-Legendre spectral approach for determining FIDE via the initial conditions. In the current study, we introduced a mathematical algorithm to determine the overall equations, utilizing Gauss-collocation points and estimated the solution directly using the transmitted Legendre polynomials. The mathematical sequences presented in the preceding part confirm the efficiency of these algorithms. The answers received utilizing the proposed algorithms prove that these algorithms with a small number of transmitted Legendre polynomials provide proper results by using, we can obtain approximations with little computation efforts. 


\section{Reference}

1. Chow TS. Fractional dynamics of interfaces between soft-nanoparticles and rough substrates. Physics Letter A. 2005; 342(1-2):148-55.

2. Ortigueira M. Introduction to fraction linear systems. Part 2: discrete-time case. IEE Proc., Vis. Image Signal Process. 2000; 147(1):7-78.

3. Wittayakiattilerd W, Chonwerayuth A. Fractional Integro-Differential Equations of Mixed Type with Solution Operator and Optimal Controls. Journal of Mathematics Research. 2011; 3(3):140.

4. Sajeer K, Rodrigues P. Novel Approach of Implementing Speech Recognition using Neural Networks for Information Retrieval. Indian Journal of Science \& Technology. 2015; 8(33):34-56.

5. Rawashdeh EA. Numerical solution of fractional integro-differential equations by collocation method. Applied Mathematics and Computation. 2006; 176(1):1-6.

6. Huang XL, Yulin Z, Xiang-Yang D. Approximate solution of fractional integro-differential equations by Taylor expansion method. Computers and Mathematics with Applications. 2011; 62(3):1112-34.

7. Xindong Z, Bo T, Yinnian H. Homotopy analysis method for higher-order fractional integro-differential equations. Computers and Mathematics with Applications. 2011; 62(8):3194-203.
8. Shaher M, Muhammad A. Numerical methods for fourth order fractional integro-differential equations. Applied Mathematics and Computation. 2006; 182(1):754-60.

9. Li Y. Solving a nonlinear fractional differential equation using Chebyshev wavelets Commun. Nonlinear Sci. Numer. Simulat. 2010; 15(9):2284-92.

10. Doha EH, Bhrawy AH, Ezz-Eldien SS. A shifted Legendre spectral method for fractional-order multi-point boundary value problems. Advances in Difference Equations. 2012; 34:34-67.

11. Doha EH, Bhrawy AH, Ezz-Eldien SS. Efficient Chebyshev spectral methods for solving multi-term fractional orders differential equations. Applied Mathematical Modeling. 2011; 35:5662-72.

12. Saadatmandi A, Dehghan M. A new operational matrix for solving fractional-order differential equations. Signal Process. 2006; 84:2602-10.

13. Khader M, Sweilam H. A Chebyshev pseudo-spectral method for solving fractional order integro-differential equation. The ANZIAM Journal of Australian Mathematical Society. 2010; 51:464-75.

14. Karimi S, Ataei A. Operational Tau approximation for a general class of fractional integro-differential equations. J. Comp. and Appl. Math. 2012; 30:655-74. 